\title{
The CFTR polymorphisms poly-T, TG-repeats and M470V in Chinese males with congenital bilateral absence of the vas deferens
}

\begin{abstract}
Wu-Hua Ni ${ }^{1, *}$, Lei Jiang ${ }^{2, *}$, Qian-Jin Fei ${ }^{1}$, Jian-Yuan Jin ${ }^{1}$, Xu Yang ${ }^{1}$ and Xue-Feng Huang ${ }^{1}$
Congenital bilateral absence of the vas deferens (CBAVD) is a frequent cause of obstructive azoospermia, and mutations of the cystic fibrosis transmembrane conductance regulator (CFTR) gene have also been frequently identified in patients with CBAVD. However, the distribution of the CFTR polymorphisms M470V, poly-T, TG-repeats and F508del mutation in the Chinese CBAVD population with presumed low cystic fibrosis (CF) frequency remains to be evaluated. Samples obtained from 109 Chinese infertile males with CBAVD and 104 normal controls were analyzed for the presence of CFTR (TG)m(T)n, M470V and F508del by PCR amplification followed by direct sequencing. Our study showed that the F508del mutation was not found in our patients. The $5 \mathrm{~T}$ mutation was present with high frequency in Chinese CBAVD patients and IVS8-5T linked to either 12 or 13 TG repeats was highly prevalent among CBAVD patients (97.22\% of 72 cases and $96.91 \%$ of 97 alleles with IVS8-5T). Moreover, a statistically significant relationship between

TG12-5T-V470 haplotype and CBAVD was detected. This study indicated that the CFTR polymorphisms poly-T, TG-repeats and M470V might affect the process of CBAVD in the Chinese population.
\end{abstract}

Asian Journal of Andrology (2012) 14, 687-690; doi:10.1038/aja.2012.43; published online 30 July 2012

Keywords: CFTR; congenital bilateral absence of the vas deferens; IVS8-5T; male infertility; M470V

\section{INTRODUCTION}

Cystic fibrosis (CF) is one of the most common autosomal recessive diseases among the Caucasian population and is caused by mutations in the cystic fibrosis transmembrane conductance regulator (CFTR) gene. ${ }^{1}$ It has an incidence of $1: 2500$ live births among Caucasians. It is estimated that about one in 20-25 Caucasians carries a mutation of the CFTR gene. ${ }^{2}$ In contrast, CF is very rare in Asian populations including Chinese. ${ }^{3-5}$ However, congenital bilateral absence of the vas deferens (CBAVD) is not uncommon in Asian populations. ${ }^{6}$ CBAVD accounts for approximately $6 \%$ of cases of obstructive azoospermia ${ }^{7}$ and is responsible for $1 \%-2 \%$ of all infertility in males. ${ }^{6}$ It is well known that CBAVD is also present in nearly $95 \%$ of all CF males. ${ }^{6}$ To date, more than 1000 mutations have been identified in the CFTR gene in classic and atypical CF patients worldwide. ${ }^{8}$ Mutations of the CFTR gene have also been frequently reported in patients with CBAVD. ${ }^{6}$ In the majority of cases, CBAVD can be considered to be an atypical, genital phenotypic presentation of $\mathrm{CF}$, presenting without other clinical manifestations of CF. ${ }^{9}$

The 5T allele, R117H and F508del, are the most common CFTR mutations in Caucasian CBAVD patients. ${ }^{2}$ The poly(T) sequence located in the splicing acceptor site of intron 8 (IVS8 poly(T)) has three variants, with five, seven or nine thymidines (the 5T, 7T and 9T alleles, respectively). ${ }^{10}$ The $7 \mathrm{~T}$ or $9 \mathrm{~T}$ allele generate a predominantly normal mRNA transcript, whereas the 5T variant affects splicing efficiency and results in reduced levels of normal mRNA due to deletion of exon 9. ${ }^{11}$ The protein product of the CFTR transcript lacking exon 9 is devoid of cyclic adenosine monophosphate-activated chloride conductance, and therefore, the $5 \mathrm{~T}$ allele is now considered as a mild mutation with an incomplete penetrance. ${ }^{11,12}$ Prior studies have demonstrated that the disease penetrance of 5T depends on the copy number of its adjacent TG repeats. ${ }^{13,14}$ The number of TG repeats immediately adjacent to $5 \mathrm{~T}$ is associated with a variable efficiency of exon 9 splicing. ${ }^{15,16}$ The different alleles at (TG)m(T)n polymorphic loci at the $3^{\prime}$ end of human CFTR intron 8 determine the exon 9 splicing efficiency. ${ }^{17,18}$

In other respects, the M470V (1540A/G in exon 10) polymorphism is one of the most common polymorphisms in the CFTR gene. ${ }^{19} \mathrm{By}$ in vitro studies, it was shown that the CFTR gene carrying the $\mathrm{V}$ allele yielded a lower functional CFTR protein rate than those carrying the $\mathrm{M}$ allele, independently of the intron 8 Tn genotype. ${ }^{15}$ de Meeus et al. ${ }^{19}$ reported that strong linkage disequilibrium was observed between the 5T allele and the $\mathrm{V}$ allele of the M470V polymorphism in the CBAVD population, but not in the normal population.

In China, the 5T allele, F508del mutation and M470V polymorphism in the CBAVD population remain to be evaluated. In the present study, we have analyzed the distribution of the CFTR polymorphism M470V, poly-T, TG-repeats and F508del mutation in 109 Chinese infertile males with CBAVD and 104 normal controls. The aim of this

${ }^{1}$ Reproductive Medicine Center, The First Affiliated Hospital of Wenzhou Medical College, Wenzhou 325000, China and ${ }^{2}$ Laboratory of Internal Medicine, The First Affiliated Hospital of Wenzhou Medical College, Wenzhou 325000, China

* These authors contributed equally to this work.

Correspondence: XF Huang (xuefhuang@hotmail.com)

Received: 3 March 2012; Revised: 12 April 2012; Accepted: 2 May 2012; Published online: 30 July 2012 
study was to explore the role of CFTR gene polymorphisms or mutations in the occurrence of patients with CBAVD in the Chinese population.

\section{MATERIALS AND METHODS \\ Samples}

Blood samples were collected from 109 unrelated Chinese males with CBAVD at the Reproductive Medicine Center, The First Affiliated Hospital of Wenzhou Medical College, China, with informed consent. The diagnoses of CBAVD were initially suggested by palpable scrotal vas on physical examination and transabdominal/rectal ultrasonography and subsequently confirmed by cytobiochemical characteristics in accordance with the World Health Organization criteria (World Health Organization, 1999). Each patient had a zero sperm count. Clinical examination for CF symptoms was performed for each patient. However, no classic CF symptoms were identified in any of the patients. As controls, 104 healthy subjects with normal routine sperm parameters from the general population in East China (Zhejiang province) were also evaluated for CFTR gene polymorphisms.

\section{Analyses of CFTR gene polymorphisms}

Genomic DNA was isolated from peripheral blood using the Gentra Puregene Blood Kit (Qiagen, Hilden, Germany). The intron 8-exon 9 junction and exon 9 region that contain the polymorphic polythymidine tract and polymorphic TG dinucleotide repeats were PCRamplified with primers E9-F (5'-CAT AAA ACA AGC ATC TAT TG-3') and E9-R ( $5^{\prime}$-AGA GAC ATG GAC ACC AAA TT- $\left.3^{\prime}\right),{ }^{6}$ and these PCR products were then sequenced to determine the length of the IVS8 poly(T) and TG repeats. Detection of the M470V polymorphism and F508del was carried out by PCR amplification of exon 10 with primers E10-F ( $5^{\prime}$-GCA GAG TAC CTG AAA CAG GA-3') and E10-R (5'-CAT TCA CAG TAG CTT ACC CA- $\left.3^{\prime}\right),{ }^{20}$ and followed by direct sequencing.

\section{Statistical analyses}

Differences between percentages were tested with the chi-square statistics or Fisher's exact test. $P$ values of less than 0.05 were considered to be of statistical significance.

\section{RESULTS}

The study of IVS8 poly(T) splicing variants revealed 72 out of 109 $(66.06 \%)$ cases with the 5T variant, 25 out of $109(22.94 \%)$ with homozygous (5T/5T) and 47 of $109(43.12 \%)$ with heterozygous (5T/-). Normal controls showed 24 out of $104(23.08 \%)$ cases with the 5T variant, four $(3.85 \%)$ cases with a homozygous (5T/5T) and 20 $(19.23 \%)$ cases with a heterozygous 5 T variant (Table 1). In CBAVD patients, the frequency of T5 allele was significantly increased when compared to control population. Overall, the frequency of the $5 \mathrm{~T}$ allele was observed $97 / 218(44.50 \%)$ in the 109 CBAVD patients $(218$ alleles), whereas $28 / 208(13.46 \%)$ in the 104 control males (208 alleles).

A significant 19 fold increase in TG13 frequency (Table 2) was observed in CBAVD patients. The frequency of TG12 allele was also statistically significantly higher in patients with CBAVD (55.05\%) than in the control males (44.23\%). The frequency of TG13 allele was observed 20/218 (9.17\%) in the 109 CBAVD patients (218 alleles) and $1 / 208(0.48 \%)$ in the control males, whereas the frequency of the TG11 allele $(35.78 \%)$ was significantly decreased in the CBAVD patients in comparison with controls $(55.29 \% ; P<0.0001)$.
Table 1 The frequency of (T)n polymorphism of intron 8 alleles in Chinese CBAVD and controls

\begin{tabular}{lcc}
\hline \multicolumn{3}{c}{ Alleles } \\
\cline { 2 - 3 } & Chinese CBAVD $(\mathrm{n}=109) *$ & Chinese controls $(\mathrm{n}=104)$ \\
\hline 5T & $97 / 218(44.50 \%)$ & $28 / 208(13.46 \%)$ \\
7T & $121 / 218(55.50 \%)$ & $179 / 208(86.06 \%)$ \\
9T & $0 / 218(0 \%)$ & $1 / 208(0.48 \%)$ \\
& Population frequency \\
5T/5T & $25 / 109(22.94 \%)$ & $4 / 104(3.85 \%)$ \\
5T/- & $47 / 109(43.12 \%)$ & $20 / 104(19.23 \%)$ \\
\hline
\end{tabular}

Abbreviation: CBAVD, congenital bilateral absence of the vas deferens. $* P<0.0001$ vs. the normal control group.

M470V in Chinese infertile males with CBAVD is not significantly different from that in the control males (Table 3). The mutation F508del, the most frequent mutation reported in CBAVD infertile males from the European and American populations, was not found in all Chinese males with CBAVD and controls.

The comparison of TG-T haplotypes revealed the significant 2.5-fold increase of the TG12-5T haplotype in males with CBAVD (Table 4). TG11-5T and TG13-5T haplotypes were found 3/218 $(1.38 \%)$ and $20 / 218(9.17 \%)$ in the CBAVD patients and were not found in the control males. However, significant increases of TG117T and TG12-7T haplotypes were observed in the control males (Table 4). One case with the TG13-7T and TG12-9T genotype was found in the control males. To the best of our knowledge, the haplotypes (TG13-7T and TG12-9T) were never reported previously. The TG12-5T haplotype was found in $33.94 \%$ of CBAVD patients and $76.29 \%$ of CBAVD patients with IV8-5T. Our finding suggests that the haplotype TG12-5T might be the most common disease-associated combination and impair the process of CBAVD.

Since the haplotype TG12-5T was found 81/96 (84.38\%) in males carrying the 5T allele (57/72 (79.17\%) in CBAVD and 24/24 (100\%) in normal controls), the association of TG12-5T and M470V was analyzed in CBAVD patients and normal controls (Table 5). Statistical analysis showed that the TG12-5T-V470 genotype was significantly associated with CBAVD (30/57 or $52.63 \%$ ) as compared to normal controls $(5 / 24$ or $20.83 \%) \quad\left(\chi^{2}=6.959, \quad P<0.01 ; \quad \mathrm{OR}=4.22\right.$, $95 \% \mathrm{CI}=1.39-12.86)$.

\section{DISCUSSION}

CBAVD is characterized by bilateral absence of the vas deferens that leads to azoospermia and male infertility. ${ }^{21}$ It accounts for $6 \%$ of cases of obstructive azoospermia. ${ }^{7}$ On the other hand, a large number of reports have described the CFTR mutation in infertile patients with CBAVD, but their results were not completely consistent. ${ }^{22}$ The main objectives of this study were to determine the possible involvement of CFTR dysfunction in CBAVD males from China, a country with a

Table 2 The frequency of (TG)m Polymorphism of intron 8 alleles in Chinese CBAVD and controls

\begin{tabular}{lcc}
\hline & \multicolumn{2}{c}{ Alleles } \\
\cline { 2 - 3 } & Chinese CBAVD $(\mathrm{n}=109) *$ & Chinese controls $(\mathrm{n}=104)$ \\
\hline TG11 & $78 / 218(35.78 \%)$ & $115 / 208(55.29 \%)$ \\
TG12 & $120 / 218(55.05 \%)$ & $92 / 208(44.23 \%)$ \\
TG13 & $20 / 218(9.17 \%)$ & $1 / 208(0.48 \%)$ \\
\hline
\end{tabular}

Abbreviation: CBAVD, congenital bilateral absence of the vas deferens. $* P<0.0001$ vs. the normal control group. 
Table 3 The distribution of the M470V allele in Chinese CBAVD and controls

\begin{tabular}{|c|c|c|}
\hline & Chinese CBAVD $(\mathrm{n}=109) *$ & Chinese controls $(n=104)$ \\
\hline \multicolumn{3}{|c|}{ Genotype distribution } \\
\hline $\mathrm{M} / \mathrm{M}$ & $30 / 109(27.52 \%)$ & 19/104 (18.27\%) \\
\hline $\mathrm{M} / \mathrm{N}$ & $42 / 109(38.53 \%)$ & 50/104 (48.08\%) \\
\hline $\mathrm{V} / \mathrm{V}$ & $37 / 109(33.94 \%)$ & $35 / 104(33.65 \%)$ \\
\hline \multicolumn{3}{|c|}{ Allele frequencies } \\
\hline M470 & 102/218 (46.79\%) & $88 / 208$ (42.31\%) \\
\hline V470 & $116 / 218$ (53.21\%) & $120 / 208$ (57.69\%) \\
\hline
\end{tabular}

Abbreviation: CBAVD, congenital bilateral absence of the vas deferens. $* P>0.05$ vs. the normal control group.

presumed low frequency of CF. We analyzed the CFTR gene in 109 Chinese CBAVD patients for the genotype of TG-repeat and polythymidine tract $(\mathrm{TG}) \mathrm{m}(\mathrm{T}) \mathrm{n}$ in intron $8, \mathrm{M} 470 \mathrm{~V}$ and F508del in exon 10. The $5 \mathrm{~T}$ variant in the intron 8 polythymidine tract was the most frequent CFTR gene alteration identified in Caucasian individuals with CBAVD. ${ }^{23}$ This nonfunctional CFTR mRNA accounts for up to $92 \%$ of the total mRNA when the $5 \mathrm{~T}$ allele is found on both CFTR genes. ${ }^{11}$ We observed a significant proportion of Chinese CBAVD males who have the $5 \mathrm{~T}$ allele $(44.50 \%)$, as compared with the control males (13.46\%). Also, a high frequency of homozygous (5T/5T) was found in the Chinese CBAVD patients (Table 1). The frequency of IVS8-5T in our patients was higher than in Portuguese $(27.4 \%),{ }^{24}$ Iranian $(25.94 \%),{ }^{25}$ Chinese residents of Taiwan $(29.2 \%)^{26}$ and Turkish patients $(19.6 \%){ }^{27}$ The compound heterozygote with a major mutation of CFTR such as F508del or R117H, which causes the development of CBAVD in Caucasian populations, determined the pathogenic $5 \mathrm{~T}$ allele. ${ }^{10}$ However, no major CFTR mutations, such as F508del, were found in Japanese and Chinese residents of Taiwan subjects with CBAVD. ${ }^{6}$ In our study, we also found no F508del mutation in Chinese population with CBAVD. Therefore, our results suggest that this allele may not be a critical mutation in the Chinese CBAVD population.

Previous studies suggested that IVS8-5T shows higher disease penetrance when it is combined with the higher number of IVS8-(TG)m. ${ }^{15}$ It was found that $5 \mathrm{~T}$ was in cis with three different TG repeats (TG115T, TG12-5T, TG13-5T). ${ }^{13}$ In 72 cases with 97 alleles of IVS8-5T present in 109 Chinese CBAVD patients, 70 cases (97.22\%) and 94 alleles $(96.91 \%)$ had 12 or 13 TG repeats adjacent to 5 T. TG12-5T was the most common disease-associated combination, and was found in $33.94 \%$ of the Chinese CBAVD patients versus $13.46 \%$ of controls (Table 4). It is interesting to note that TG11-5T and TG13-5T were found exclusively in the Chinese CBAVD patients. Prior studies have

Table 4 The frequency of (TG)m(T)n haplotypes in Chinese CBAVD and controls

\begin{tabular}{lcc}
\hline \multirow{2}{*}{$\begin{array}{l}\text { Linkage } \\
\text { haplotypes }\end{array}$} & \multicolumn{2}{c}{ Alleles } \\
\cline { 2 - 3 } & Chinese CBAVD $(\mathrm{n}=109) *$ & Chinese controls $(\mathrm{n}=104)$ \\
\hline TG11-5T & $3 / 218(1.38 \%)$ & $0 / 208(0 \%)$ \\
TG12-5T & $74 / 218(33.94 \%)$ & $0 / 208(0 \%)$ \\
TG13-5T & $20 / 218(9.17 \%)$ & $115 / 208(55.29 \%)$ \\
TG11-7T & $75 / 218(34.40 \%)$ & $63 / 208(30.29 \%)$ \\
TG12-7T & $46 / 218(21.10 \%)$ & $1 / 208(0.48 \%)$ \\
TG13-7T & $0 / 172(0 \%)$ & $1 / 208(0.48 \%)$ \\
TG12-9T & $0 / 172(0 \%)$ &
\end{tabular}

Abbreviation: CBAVD, congenital bilateral absence of the vas deferens.

$* P<0.0001$ vs. the normal control group.
Table 5 The association of TG12-5T and M470V in Chinese CBAVD and controls

\begin{tabular}{|c|c|c|c|}
\hline \multirow{2}{*}{ Phenotype } & \multicolumn{3}{|c|}{ M470V } \\
\hline & M470/M470 & M470/V470 & V470/V470 \\
\hline Chinese CBAVD (TG12-5T) & 2/57 (3.51\%) & $25 / 57(43.86 \%)$ & $30 / 57(52.63 \%)$ \\
\hline Chinese controls (TG12-5T) & $2 / 24(8.33 \%)$ & 17/24 (70.83\%) & $5 / 24(20.83 \%)$ \\
\hline
\end{tabular}

Abbreviation: CBAVD, congenital bilateral absence of the vas deferens.

demonstrated that a $5 \mathrm{~T}$ variant, along with the preceding higher repetitive dinucleotide TG sequences such as (TG) 13 or (TG)12, produces more mRNA transcripts with inframe deletion of exon $9 .{ }^{11,15,28}$ Groman et al. ${ }^{13}$ suggested that TG repeat number is a reliable predictor of penetrance for pathogenic $5 \mathrm{~T}$ alleles. Those individuals carrying TG12-5T or TG13-5T were substantially more likely to exhibit an abnormal phenotype than those carrying TG11-5T. ${ }^{13}$ TG11-5T was by far the most common in United States, Czech Republic, Poland, Italy and Germany populations, followed by TG12-5T and TG13-5T. ${ }^{13}$ However, our study showed that TG11-5T was not found in the normal Chinese population (Table 4). Ethnic differences in the incidence of CFTR (TG) $\mathrm{m}(\mathrm{T}) \mathrm{n}$ around the world are quite striking. ${ }^{14}$

Another common variant in CFTR, a methionine or valine at codon 470 , was suggested to influence the penetrance of $5 \mathrm{~T} .{ }^{15}$ The quantity and quality of CFTR transcripts and/or proteins was affected by (TG) $\mathrm{m}(\mathrm{T}) \mathrm{n}$ and M470V polymorphic loci. ${ }^{15}$ Previous studies have demonstrated that the 5T-V470 haplotype showed a higher disease association than the 5T-M470 haplotype. ${ }^{19,25}$ Cuppens et al. ${ }^{15}$ demonstrated that the V470 allele yielded a lower functional CFTR protein rate than the M470 allele, independently of the intron 8 genotypes, using in vitro studies. In the present study, the $\mathrm{V}$ allele frequency is not increased in the Chinese CBAVD population (Table 3). However, we found a significant association between the TG12-5T-V470 haplotype and CBAVD (Table 5), in agreement with a previous report. ${ }^{29}$ Thus, although a particular allele by itself might have no deleterious consequences, the combination of specific alleles at several polymorphic loci might lead to less functional or even insufficient CFTR protein. ${ }^{15,30}$

In conclusion, this study indicates that the 5T allele in intron 8 of CFTR or IVS8-5T linked to either 12 or 13 TG repeats exhibit a high prevalence among the CBAVD patients tested. Moreover, a statistically significant relationship between the TG12-5T-V470 haplotype and CBAVD was detected for the Chinese population. These data provide a better characterization of CBAVD patients in China. However, the search for other mutations in the CFTR gene should be continued. There are many possible mutations to consider for analysis as more than 1000 mutations in the CFTR gene are documented in the literature (Cystic Fibrosis Mutation Database: http:// www.genet.sickkids.on.ca/cftr). ${ }^{31,32}$ The $5 \mathrm{~T}$ allele is associated with different phenotype, which is presumably due to variable penetrance. ${ }^{31}$ CBAVD patient carriers of the $5 \mathrm{~T}$ allele are more likely to have a severe or a mild mutation in the second allele. ${ }^{33}$ The combination of a severe mutation on one chromosome and a $5 \mathrm{~T}$ variant on the other seems to be one of the major causes of CBAVD. ${ }^{33}$ Clinically, intracytoplasmic single sperm injection can be used to provide fertility for men with cystic fibrosis or CBAVD. ${ }^{34}$ However, the CFTR mutation can be transmitted to the next generation by intracytoplasmic single sperm injection, even in a population with a low prevalence of $\mathrm{CF},{ }^{35}$ so the risk of having children with $\mathrm{CF}$ or CBAVD will be increased. ${ }^{33}$ At present, a careful clinical examination of children seems to be mandatory when the father has CBAVD. The major reasons for this are derived from the variable phenotypic expression of 
combinations of CFTR genes and the fact that CFTR mutations may have remained undetected in these couples. ${ }^{33}$ Cystic fibrosis mutation analysis is required in CBAVD patients and their partners. ${ }^{36}$ Thus, further counseling is suggested for patients with CBAVD, or with a family history of CBAVD. ${ }^{37}$

\section{AUTHOR CONTRIBUTIONS}

WHN carried out the molecular genetic studies, participated in data analysis and drafted the manuscript. LJ participated in the sequence alignment and designed of the study and drafted the manuscript. QJF participated in the patients sample collection and data analysis, JYJ participated in the sequence alignment and patients sample collection. $\mathrm{XY}$ participated in the patients sample collection and data analysis, $\mathrm{XFH}$ conceived of the study, participated in its design and coordination and helped to draft the manuscript. All authors read and approved the final manuscript.

\section{COMPETING FINANCIAL INTERESTS}

All authors declare that there are no competing financial interests.

\section{ACKNOWLEDGMENTS}

The Project Supported by Wenzhou Science and Technology Bureau Program (No. Y20110124).

1 Zielenski J, Tsui LC. Cystic fibrosis: genotypic and phenotypic variations. Annu Rev Genet 1995; 29: 777-807.

2 Grangeia A, Niel F, Carvalho F, Fernandes S, Ardalan A et al. Characterization of cystic fibrosis conductance transmembrane regulator gene mutations and IVS 8 poly(T) variants in Portuguese patients with congenital absence of the vas deferens. Hum Reprod 2004; 19: 2502-8.

3 Li N, Pei P, Bu DF, He B, Wang GF. A novel CFTR mutation found in a Chinese patient with cystic fibrosis. Chin Med J (Engl) 2006; 119: 103-9

4 Alper OM, Shu SG, Lee MH, Wang BT, Lo SY et al. Detection of novel CFTR mutations in Taiwanese cystic fibrosis patients. J Formos Med Assoc 2003; 102: 287-91.

5 Wong LJ, Alper OM, Wang BT, Lee MH, Lo SY. Two novel null mutations in a Taiwanese cystic fibrosis patient and a survey of East Asian CFTR mutations. Am J Med Genet $A$ 2003; 120A: 296-8

6 Anzai C, Morokawa N, Okada H, Kamidono S, Eto $\mathrm{Y}$ et al. CFTR gene mutations in Japanese individuals with congenital bilateral absence of the vas deferens. J Cyst Fibros 2003; 2 : 14-8.

7 Jequier AM, Ansell ID, Bullimore NJ. Congenital absence of the vasa deferentia presenting with infertility. J Androl 1985; 6: 15-9.

8 Chen JM, Cutler C, Jacques C, Boeuf G, Denamur $\mathrm{E}$ et al. A combined analysis of the cystic fibrosis transmembrane conductance regulator: implications for structure and disease models. Mol Biol Evol 2001; 18: 1771-88.

9 Timmreck LS, Gray MR, Handelin B, Allito B, Rohlfs E et al. Analysis of cystic fibrosis transmembrane conductance regulator gene mutations in patients with congenital absence of the uterus and vagina. Am J Med Genet A .2003; 120A: 72-6

10 Kiesewetter S, Macek M Jr, Davis C, Curristin SM, Chu CS et al. A mutation in CFTR produces different phenotypes depending on chromosomal background. Nat Genet 1993; 5: 274-8.

11 Chu CS, Trapnell BC, Curristin S, Cutting GR, Crystal RG. Genetic basis of variable exon 9 skipping in cystic fibrosis transmembrane conductance regulator mRNA. Nat Genet 1993; 3: 151-6.

12 Teng H, Jorissen M, van Poppel H, Legius E, Cassiman JJ et al. Increased proportion of exon 9 alternatively spliced CFTR transcripts in vas deferens compared with nasal epithelial cells. Hum Mol Genet 1997; 6: 85-90.

13 Groman JD, Hefferon TW, Casals T, Bassas L, Estivill X et al. Variation in a repeat sequence determines whether a common variant of the cystic fibrosis transmembrane conductance regulator gene is pathogenic or benign. Am J Hum Genet 2004; 74: $176-9$.
14 Chiang HS, Lu JF, Liu CH, Wu YN, Wu CC. CFTR (TG)m(T)n polymorphism in patients with CBAVD in a population expressing low incidence of cystic fibrosis. Clin Genet 2009; 76: 282-6.

15 Cuppens $\mathrm{H}$, Lin W, Jaspers M, Costes B, Teng H et al. Polyvariant mutant cystic fibrosis transmembrane conductance regulator genes. The polymorphic $(\mathrm{Tg}) \mathrm{m}$ locus explains the partial penetrance of the T5 polymorphism as a disease mutation. J Clin Invest 1998; 101: 487-96.

16 Niksic M, Romano M, Buratti E, Pagani F, Baralle FE. Functional analysis of cis-acting elements regulating the alternative splicing of human CFTR exon 9. Hum Mol Genet 1999; 8: 2339-49.

17 Pagani F, Buratti E, Stuani C, Romano M, Zuccato E et al. Splicing factors induce cystic fibrosis transmembrane regulator exon 9 skipping through a nonevolutionary conserved intronic element. J Biol Chem 2000; 275: 21041-7.

18 Radpour R, Gourabi H, Gilani MA, Dizaj AV. Molecular study of (TG)m(T)n polymorphisms in Iranian males with congenital bilateral absence of the vas deferens. J Androl 2007; 28: 541-7.

19 de Meeus A, Guittard C, Desgeorges M, Carles S, Demaille J et al. Linkage disequilibrium between the M470V variant and the IVS8 polyT alleles of the CFTR gene in CBAVD. J Med Genet 1998; 35: 594-6.

20 Wu CC, Alper OM, Lu JF, Wang SP, Guo L et al. Mutation spectrum of the CFTR gene in Taiwanese patients with congenital bilateral absence of the vas deferens. Hum Reprod $2005 ; 20: 2470-5$

21 Uzun S, Gokce S, Wagner K. Cystic fibrosis transmembrane conductance regulator gene mutations in infertile males with congenital bilateral absence of the vas deferens. Tohoku J Exp Med 2005; 207: 279-85.

$22 \mathrm{Yu} \mathrm{J}, \mathrm{Chen} \mathrm{Z,} \mathrm{Ni} \mathrm{Y,} \mathrm{Li} \mathrm{Z.} \mathrm{CFTR} \mathrm{mutations} \mathrm{in} \mathrm{men} \mathrm{with} \mathrm{congenital} \mathrm{bilateral} \mathrm{absence} \mathrm{of}$ the vas deferens (CBAVD): a systemic review and meta-analysis. Hum Reprod 2012; 27: 25-35.

23 Chillon M, Casals T, Mercier B, Bassas L, Lissens W et al. Mutations in the cystic fibrosis gene in patients with congenital absence of the vas deferens. N Engl J Med 1995; 332: 1475-80.

24 Grangeia A, Carvalho F, Fernandes S, Silva J, Sousa M et al. A novel missense mutation P1290S at exon-20 of the CFTR gene in a Portuguese patient with congenital bilateral absence of the vas deferens. Fertil Steril 2005; 83: 448-51.

25 Radpour R, Gilani MA, Gourabi H, Dizaj AV, Mollamohamadi S. Molecular analysis of the IVS8-T splice variant 5T and M470V exon 10 missense polymorphism in Iranian males with congenital bilateral absence of the vas deferens. Mol Hum Reprod 2006; 12: 469-73.

26 Wu CC, Hsieh-Li HM, Lin YM, Chiang HS. Cystic fibrosis transmembrane conductance regulator gene screening and clinical correlation in Taiwanese males with congenital bilateral absence of the vas deferens. Hum Reprod 2004; 19: 250-3.

27 Dayangac D, Erdem H, Yilmaz E, Sahin A, Sohn C et al. Mutations of the CFTR gene in Turkish patients with congenital bilateral absence of the vas deferens. Hum Reprod 2004; 19: 1094-100.

28 Chu CS, Trapnell BC, Murtagh JJ Jr, Moss J, Dalemans W et al. Variable deletion of exon 9 coding sequences in cystic fibrosis transmembrane conductance regulator gene mRNA transcripts in normal bronchial epithelium. EMBO J 1991; 10: 1355-63.

29 Stuppia L, Antonucci I, Binni F, Brandi A, Grifone $\mathrm{N}$ et al. Screening of mutations in the CFTR gene in 1195 couples entering assisted reproduction technique programs. Eur J Hum Genet 2005; 13: 959-64

30 Pallares-Ruiz N, Carles S, des Georges M, Guittard C, Arnal F et al. Complete mutational screening of the cystic fibrosis transmembrane conductance regulator gene: cystic fibrosis mutations are not involved in healthy men with reduced sperm quality. Hum Reprod 1999; 14: 3035-40.

31 Danziger KL, Black LD, Keiles SB, Kammesheidt A, Turek PJ. Improved detection of cystic fibrosis mutations in infertility patients with DNA sequence analysis. Hum Reprod 2004; 19: 540-6.

32 Kosova G, Pickrell JK, Kelley JL, McArdle PF, Shuldiner AR et al. The CFTR Met 470 allele is associated with lower birth rates in fertile men from a population isolate. PLOS Genet 2010; 6: e1000974

33 Lissens W, Mercier B, Tournaye $\mathrm{H}$, Bonduelle $\mathrm{M}$, Ferec $\mathrm{C}$ et al Cystic fibrosis and infertility caused by congenital bilateral absence of the vas deferens and related clinical entities. Hum Reprod 1996; 11Suppl 4: 55-78; discussion 9-80.

34 Halliday J. Outcomes for offspring of men having ICSI for male factor infertility. Asian J Androl 2012; 14: 116-20.

35 Chiang HS, Wu CC, Wu YN, Lu JF, Lin GH et al. CFTR mutation analysis of a Caucasian father with congenital bilateral absence of vas deferens, a Taiwanese mother, and twins resulting from ICSI procedure. J Formos Med Assoc 2008; 107: 736-40.

36 Oates R. Evaluation of the azoospermic male. Asian J Androl 2012; 14: 82-7.

37 Cottin V, Thibout Y, Bey-Omar F, Durieu I, Laoust L et al. Late CF caused by homozygous IVS8-5T CFTR polymorphism. Thorax 2005; 60: 974-5. 